\title{
La utilidad del propóleo en las ciencias de la salud
}

\section{Artículo de revisión}

Rodríguez-Ramírez José Félix*, Delgado-Íñiguez Fabián*, De Velasco-Herrera Paris*, Navarro-Hernández Elizabeth*, Madrigal-Elizalde Emmanuel*, Robles-Alvarez Víctor Manuel*, Martín del Campo-Galván José Israel*, Landeros-Orea Rubén**, Robledo-González María Guadalupe ${ }^{* * *}$, Casilla Peñuelas Rafael Alejandro ${ }^{* * *}$, Masuoka Ito David ${ }^{* * * *}$

\section{Resumen}

- El propóleo tiene una gran serie de propiedades biológicas y

- farmacológicas. Sus mecanismos de acción han sido amplia-

- mente estudiados en los últimos años, utilizando diferentes

- modelos experimentales in vitro e in vivo. Los investigadores

- han estado interesados en el estudio de los compuestos ais-

- lados responsables de la acción del propóleo, sin embargo, se

- carece de investigación clínica suficiente sobre sus efectos. El

- objetivo de esta revisión es analizar el potencial del propóleo

- para el desarrollo de nuevos medicamentos, utilizando los

- datos de la literatura existentes. LUX MÉDICA, AÑO 9, NÚMERO

- 26, ENERO-ABRIL 2014, PP 29-36.

-

Palabras clave: propóleo, medicamentos, propiedades

-

. biológicas

\section{Abstract}

Propolis has a large number of biological and pharmacological properties. Their mechanisms of action have been widely investigated in recent years, using different experimental models in vitro and in vivo. Researchers have been interested in the study of the isolated compounds responsible for the action of propolis. However, there is no clinical research on its effects. The objective of this review is to analyze the potential of propolis for the development of new drugs, using data from the existing literature. LUX MÉDICA, AÑO 9, NÚMERO 26, ENERO-ABRIL 2014, PP 29-36.

Key Words: propolis, drugs, biological properties

\section{Introducción}

Los productos naturales han sido utilizados con fines medicinales en la cultura popular de todo el mundo durante miles de años. En las últimas décadas, se han publicado diversos estudios donde se habla de la composición química y de las propiedades biológicas de estos productos. Uno de los productos de las abejas más estudiado

Estudiantes del noveno semestre de la carrera de Medicina, Departamento de Medicina, del Centro de Ciencias de la Salud de la Universidad Autónoma de Aquascalientes

** Estudiante de la carrera de Biotecnología, Departamento de Química, Centro de Ciencias Básicas de la Universidad Autónoma de Aguascalientes.

*** Técnico de Laboratorio de Investigación, Departamento de Estomatología, Centro de Ciencias de la Salud UAA

**** Profesor investigador, Departamento de Tecnología en Alimentos. Centro de Ciencias Agropecuarias UAA

***** Profesor Investigador, Departamento de Estomatología, Centro de Ciencias de la Salud. UAA

Fecha de recibido: 20 de enero 2014

Fecha de aceptación: 10 de febrero 2014

Correspondencia: Dr. David Masuoka D.D.S. Ph.D. Profesor e Investigador Titular. Universidad Autónoma de Aguascalientes. Departamento de Estomatología, Centro de Ciencias de la Salud. Av. Aguascalientes No.904. Unidad Médico Didáctica Edif.101, Ciudad Universitaria Aguascalientes, Ags. México 20131 Tel: +52 (449) 9108438 david.masuoka@gmail.com 
ha sido el propóleo, que es una sustancia resinosa producida por exudados y brotes de plantas mezclados con cera y enzimas de las abejas. El propóleo es utilizado por las abejas para barnizar el interior de la colmena, cerrar grietas, reducir vías de accesos; sellar agujeros en sus panales, alisar las paredes internas y consolidar los componentes estructurales, protegiendo a la colonia de enfermedades y a su vez, es utilizado para recubrir los cadáveres de intrusos que murieron dentro de la colmena, evitando con esto su descomposición.

La composición química del propóleo, así como su actividad biológica, su color y su aroma depende de factores asociados a la región geográfica, la temporada de recolección, el tipo de vegetación, la especie de abeja y el solvente usado para su extracción $n^{1,2}$ .Su color varía del verde amarillento al marrón oscuro dependiendo de su origen y edad ${ }^{3}$. Es duro y quebradizo con el frío, pero se vuelve suave y muy pegajoso cuando está caliente. Se ha encontrado que su composición general está basada en $50 \%$ de resina y bálsamo vegetales, $30 \%$ de cera, 10\% de aceites esenciales y aromáticos, $5 \%$ de polen y $5 \%$ de otras sustancias diversas, incluyendo desechos orgánicos ${ }^{1,2,4}$. En cuanto a su composición química, el propóleo contiene amilasa, muchos compuestos polifenólicos, flavonoides, ácidos fenólicos, ésteres y ácidos. Por medio de electroforesis capilar de tres diferentes extractos de propóleo en etanol, etanol-acuoso y acuoso glicólico, se determinaron doce flavonoides; pinocembrina, acacetín, crisina, rutina, catequina, naringenina, galangina, luteolina, kaempferol, apigenina, miricetina y quercetina, dos ácidos fenólicos, ácido cinámico y ácido cafeico, un derivado de estilbeno los estilbenos, resveratrol ${ }^{4}$. Observando que en el extracto de propóleos acuoso-etanólico mostró un gran porcentaje de ácido cafeico, galangina, la quercetina y la crisina, mientras que la preparación de etanol se compone de una gran cantidad de resveratrol, crisina, y el ácido cafeico ${ }^{5,6}$. En el contrario, la preparación de propóleos acuoso-glicólico está compuesto por aproximadamente $11 \%$ de ácido cafeico y una baja cantidad de los otros flavonoides identificados debido a la presencia de aproximadamente $85 \%$ de los compuestos no identificados ${ }^{5,6}$.

Otro punto importante en su composición química es que el propóleo contiene algunos minerales como el $\mathrm{Mg}, \mathrm{Ca}, \mathrm{I}, \mathrm{K}, \mathrm{Na}$, $\mathrm{Cu}, \mathrm{Zn}, \mathrm{Mn}$ y $\mathrm{Fe}$, así como algunas vitaminas como la B1, B2, B6, $C$ y $E$, y una serie de ácidos grasos. Además, se contiene algunas enzimas como la deshidrogenasa succínico, la glucosa- 6-fosfata$\mathrm{sa}$, trifosfato de adenosina y el ácido fosfatasa ${ }^{5}$. Así como cobre $26,5 \mathrm{mg} / \mathrm{kg}$ y manganeso $40 \mathrm{mg} / \mathrm{kg}^{4,7}$. Los residuos de ceniza contiene hierro, calcio, aluminio, vanadio, estroncio, manganeso y silicio. 


\section{Aplicaciones clínicas del propóleo}

El propóleo presenta una serie de propiedades biológicas y farmacológicas, como inmunomodulador, antitumoral, antinflamatorio, antioxidante, antibacteriano, antiviral, antimicótico y actividad antiparasitara, entre otros ${ }^{8,9}$.

Se ha descrito que los métodos in vitro son útiles para la investigación preliminar de la posible capacidad de un producto natural. Además, los ensayos in vitro e in vivo no siempre incluyen extractos caracterizados químicamente, y se debe tener en cuenta que se puede esperar variabilidad de los preparados farmacológicos ${ }^{10}$.

Aunque varios autores han estado investigando las actividades biológicas del propóleo, no existe una revisión crítica sobre la utilidad de estos datos en el contexto del uso clínico del producto. Por otro lado, existen las nuevas formulaciones que contienen propóleo o sus compuestos aislados. A modo de ejemplo, en un estudio se prepararon micropartículas esféricas y homogéneas de poli (epsiloncaprolactona) (PCL), que contiene propóleo, con $60 \%$ de la sustancia la cual se libera en 48 horas $^{11}$. Recientemente, se ha estudiado el uso potencial de la cavidad de beta-ciclodextrina para la incorporación de componentes específicos de propóleo con el objetivo de aumentar su solubilidad en agua ${ }^{12}$. La eficiencia de los extractos etanólicos y de agua de propóleo en la síntesis de nanopartículas de Ag y Au se comparó con la del hidroxiflavonoides natural, pinocembrina y galangina aislado del propóleo; que son igualmente eficaces en la síntesis rápida y la estabilización de las nanopartículas de $\mathrm{Ag}$ y $\mathrm{Au}^{13}$. El objetivo de esta revisión es analizar la posibilidad del propóleo para el desarrollo de nuevos medicamentos en algunos campos de investigación, como la inmunología (por ejemplo, medicamentos con acción inmunomoduladora), tumores (siendo las células tumorales el blanco para el propóleo o sus compuestos aislados), infecciones (el potencial del propóleo o sus componentes como agentes cariostático y para el desarrollo de productos biotecnológicos para el control de caries y otras enfermedades infecciosas), alergia (el propóleo puede ser eficaz en el alivio de los síntomas de la rinitis alérgica), diabetes (el propóleo parece poseer efecto preventivo sobre la destrucción de células beta pancreáticas) y las úlceras (las propiedades del propóleo anti-ulcerogénico, así como sus principales ácidos fenólicos). La tabla uno presenta algunas propiedades biológicas del propóleo y los métodos experimentales utilizados por diferentes autores.

\section{Tabla I}

Comparación de estudios sobre propiedades biológicas del propóleo y los métodos experimentales utilizados

\begin{tabular}{|lccc}
\hline Propiedades Biológicas & In vitro / in vivo & Concentración de propóleo & Autores \\
\hline Inmunomodulador & In vivo & $200 \mathrm{mg} / \mathrm{kg}$ & Orsatti et al. (2010a,b) \\
\hline & In vivo & $3-300 \mu \mathrm{g} / 100 \mu \mathrm{l}$ & Orsi et al. (2005) \\
\hline Anti-tumoral & In vivo & $50 \mathrm{y} 150 \mathrm{mg} / \mathrm{kg}$ & Orsolic et al. (2005) \\
\hline & In vivo & $5-100 \mu \mathrm{g} / 100 \mu \mathrm{l}$ & Bassani Silva et al. (2007) \\
\hline Antimicrobiano & & & \\
\hline Antibacterial & In vivo & $0.4-14.0 \% \mathrm{v} / \mathrm{v}$ & Sforcin et al. (2000) \\
\hline Antimicótico & In vivo & $0.4-14.0 \% \mathrm{v} / \mathrm{v}$ & Sforcin et al. (2001) \\
\hline Antiviral & In vivo & $5-100 \mu \mathrm{g} / 100 \mu \mathrm{l}$ & Búfalo et al. (2009c) \\
\hline Anti-diabético & In vivo & 100 y $300 \mathrm{mg} / \mathrm{kg}$ & Zamami et al. (2007) \\
\hline Anti-ulceroso & In vivo & 50,250 y $500 \mathrm{mg} / \mathrm{kg}$ & Barros et al. (2007) \\
\hline
\end{tabular}


La composición química del propóleo depende de las características fitogeográficas del sitio de recolección, ya que las abejas eligen diferentes plantas como fuente de propóleo en diferentes hábitats ${ }^{14}$. Este aspecto dificulta la estandarización del propóleo, así como sus diferentes disolventes (etanol, metanol y agua), ya que pueden extraer compuestos diferentes que influyen en su actividad $^{15}$. Por lo tanto, hasta el momento una estandarización universal sería imposible. Se ha propuesto que las propiedades biológicas del propóleo deben estar vinculadas a una investigación detallada de su composición química y sus orígenes botánicos ${ }^{16}$. Siendo necesario de igual manera el estudiar la ausencia de metales pesados y pesticidas, se ha sugerido que el propóleo puede servir como un indicador para el estudio de la contaminación ambiental ${ }^{17}$. Un gran número de artículos tratan diferentes aspectos de las propiedades biológicas del propóleo y han sido publicados en las últimas décadas. Sin embargo, una parte considerable de ellos son de utilidad limitada, a pesar de que describa su actividad como "fuerte", "notable" o "significativo". La razón es la falta de base para la comparación y evaluación científica de los resultados, porque no se refieren a la naturaleza química de las muestras de propóleos estudiados, ya que estos estudios sólo informan que las pruebas se han realizado con extractos de propóleo. Siendo importante tener en cuenta que no hay tal cosa como "sólo propóleo". Aunque de origen vegetal, el propóleo es un producto de las abejas, siendo que la materia prima la obtienen de diferentes plantas en los distintos ecosistemas, por lo que es importante el seleccionar y establecer los representantes apropiados de la flora local. El término "propóleo" no tiene una connotación química a diferencia del nombre científico de una especie vegetal. Una especie de planta se caracteriza por su genoma y la genómica con el tiempo determina los metabolitos secundarios sintetizados por las enzimas de la planta y responsable de sus actividades biológicas. Por otro lado, también contiene metabolitos secundarios de plantas; sin embargo, éstas no son las mismas en todo el mundo. En la tabla dos se describen los principales tipos químicos del propóleo de acuerdo con su principal fuente herbolaria y región.

\section{Tabla 2}

\section{Los tipos de propóleo más comunes}

\begin{tabular}{|c|c|c|c|c|}
\hline Tipo de propóleo & Origen geográfico & Fuente herbolaria & Constituyentes mayores & Autores \\
\hline $\begin{array}{l}\text { Verde (alecrim) } \\
\text { Brasileño }\end{array}$ & Brazil & $\begin{array}{l}\text { Baccharisspp., } \\
\text { predominatemente } \mathrm{B} \text {. } \\
\text { dracunculifolia DC. }\end{array}$ & $\begin{array}{l}\text { Ácidos prenylated } \\
\text { p-coumaric, } \\
\text { ácidos diterpénicos. }\end{array}$ & Salatino et al. (2005) \\
\hline Birch & Russia & BetulaverrucosaEhrh & $\begin{array}{l}\text { Flavones, flavonoles } \\
\text { (distintos a los del } \\
\text { tipo Poplar) }\end{array}$ & Popravko (1978) \\
\hline Propóleo rojo & Cuba, Brazil, México & Dalbergiaspp. & $\begin{array}{l}\text { Isoflavonoides } \\
\text { (isovlavans, pterocarpans) }\end{array}$ & $\begin{array}{l}\text { Campo Fernandezet al. } \\
\text { (2008) } \\
\text { Daugsch et al. (2008) } \\
\text { Lotti et al. (2010) }\end{array}$ \\
\hline "Clusia" & Cuba, Venezuela & Clusiaspp. & Poliprenilato benzofenones & $\begin{array}{l}\text { Cuesta-Rubio et al. (2002) } \\
\text { Trusheva et al. (2004) }\end{array}$ \\
\hline "Pacífico" & $\begin{array}{l}\text { Región del Pacífico: Okinawa } \\
\text { Taiwan } \\
\text { Indonesia }\end{array}$ & Macarangatanarius & C-Prenil-flavonones & $\begin{array}{l}\text { Chen et al. (2008), } \\
\text { Kumazawa et al. (2008) } \\
\text { Trusheva et al. (en proceso) }\end{array}$ \\
\hline
\end{tabular}


Al pensar en cómo se seleccionará el propóleo con el fin de comprobar su actividad biológica, lo más importante es tratar de enfocarnos a muestras de áreas donde el propóleo nunca ha sido estudiado antes. Es muy probable que las abejas hayan encontrado una fuente de alguna planta que tenga una actividad promisoria, y que ésta tenga el potencial de proporcionar nuevos compuestos naturales biológicamente activos. En general, las abejas eligen materiales resinosos de origen vegetal por sus propiedades físicas para ser usado como el propóleo. Siendo que este material servirá como defensa química contra los microorganismos, sobre la base de su composición química ${ }^{16,18}$. Estudios comparativos han mostrado que a pesar de que su composición química pueda ser de origen distinto, el propóleo siempre ha demostrado una actividad más o menos considerablemente biológica ${ }^{5,7}$. Por esta razón, la diversidad química del propóleo tiene el potencial de proporcionar datos valiosos ${ }^{4}$. El propóleo sin refinar contiene impurezas tales como madera, cera, polen, e incluso abejas muertas, por lo que es necesario una observación macroscópica de la muestra con el fin de eliminar y purificarla antes de la preparación de sus extractos, un paso crítico en el proceso de prueba es la extracción de las muestras de propóleo que se utilizará en el estudio. Los disolventes utilizados para la extracción suele ser alcoholes como metanol y etanol. El más utilizado es el etanol que contiene disolventes con un diferente porcentaje de agua, siendo el etanol al $70 \%$ el que ha demostrado ser el idóneo para extraer la mayor parte de los componentes activos del propóleo ${ }^{19}$. Como el propóleo puede contener hasta un $20-30 \%$ de cera, este solvente se ha aplicado en muchos estudios. El agua también se ha utilizado en muchas ocasiones, sin embargo, es importante tener en cuenta que en general el agua solo di- suelve una pequeña parte de los componentes de propóleos, alrededor del 10\% de su peso, mientras que el etanol al $70 \%$ puede disolver el $50-70 \%$, sin embargo, esto dependerá de la cantidad de cera.

Los extractos de propóleos son elaborados por maceración, en algunos casos por extracción Soxhlet (con metanol o etanol al 96\%), o por extracción ultrasónica asistida, la cual parece dar excelentes resultados, ya que acelera el proceso ${ }^{6}$, por otro lado, la extracción asistida por microondas resulta ser menos favorable, especialmente en el caso de las muestras ricas en compuestos fenólicos, ya que podría conducir a la disminución del contenido fenólico, debido a los procesos de oxidación ${ }^{6}$.

En general, el perfil metabólico del extracto da una idea de su origen vegetal y permite la identificación de sus principales constituyentes, y también de una serie de elementos menores que lo componen dependiendo de la técnica. Esto nos puede revelar los tipos de compuestos presentes y da una idea de las posibles actividades que se esperan. Por ejemplo, la presencia de una cantidad significativa y el número de compuestos fenólicos podrían llevar a la expectativa de que el extracto tiene el potencial de expulsar radicales libres, y se podrían demostrar bioactividades relacionadas con este potencial.

Cualquier estudio sobre el tipo de actividad biológica del propóleo, debe comenzar con un perfil químico de los extractos que se utilizarán en el estudio. Diferentes técnicas son adecuadas para el propósito de obtener un perfil químico, como lo demuestran los numerosos artículos que tratan del análisis del propóleo. Las técnicas híbridas son las más apropiadas: HPLC-DAD, LCMS, LC-MS-MS, GC-MS. La relativa naturaleza polar de los componentes del propóleo (por lo general tienen varios grupos $\mathrm{OH}$ en su molécula), combinado con las técnicas de ionización suave compatible con la cromatografía líquida, hizo posible 
que la cromatografía líquida de alta resolución con detector de arreglo de diodos (HPLC-DAD) y la cromatografía líquida de alta resolución con espectrometría de masas (HPLC-MS), sean los métodos favoritos para el análisis de los componentes del propóleo. Sin embargo, la importancia de los capilares de la cromatografía de gases (GC) y la valiosa información estructural proporcionada por el impacto de electrones en la espectrometría de masa(EIMS) han demostrado ser todavía útiles. La utilización de la cromatografía de gases con la espectrometría de masa (GC-MS) ha tenido un notable resurgimiento, como lo demuestran varios estudios $^{20,21}$

Los datos cuantitativos son muy recomendables, pero su obtención no siempre es posible y tampoco razonable. En el caso del propóleo por sus tipos de origen químico más populares, como es el caso del propóleo de álamo europeo y el propóleo de romero verde brasileño, los componentes biológicamente activos son ampliamente conocidos y se han propuesto métodos para la estandarización y control de calidad, por lo que para el propóleo de álamo europeo, la cuantificación fenólica total, la de flavonas/flavonoles total y la deflavanones/dihidroflavonolestotal, se utilizan como una medida de la cantidad de sus principios activos ${ }^{22}$. Para el propóleo de romero verde brasileño se utiliza la cuantificación de fenoles totales y flavonoides totales ${ }^{23}$. Estos métodos son propuestos por la Comisión Internacional de la Miel. Es importante recordar que los valores característicos para el contenido de flavonoides y fenólicos son diferentes para cada tipo de propóleo, y los compuestos de referencia utilizada para la calibración también son diferentes. Por supuesto que hay muchos tipos de propóleo: en el Pacífico el que se deriva de macaranga ${ }^{24}$, en el Mediterráneo que contienen principalmente diterpenos ${ }^{25}$, en América del Sur (Cuba, Brasil, México) el llamado propóleo rojo que se deriva de la dalbergia ${ }^{26,27}$. Entre ellos se encuentran algunos con un contenido fenólico muy bajo o nulo. Para cada uno de ellos es necesario proponer métodos para su estandarización y control de calidad que todavía no se han desarrollado. Este proceso está en marcha, por ejemplo, el procedimiento propuesto recientemente para la cuantificación espectrofotométrica de flavanones total en el tipo de propóleo del Pacífico derivado de macaranga 14. La cuantificación de los componentes individuales no es necesaria en las etapas iniciales de los estudios, a medida que la investigación vaya avanzando esto podría ser significativo, especialmente si las muestras con una composición cualitativa similar demuestran diferencias significativas en sus actividades.

Las propiedades biológicas del propóleo han sido estudiadas ampliamente en los últimos años. Existe un interés aparente, que nos señala que el propóleo tiene un potencial para el desarrollo de nuevos medicamentos, atrayendo un gran interés de los consumidores en adquirir productos que contengan propóleo, los cuales son por lo general comercializados por las tiendas naturistas. Sin embargo, con el fin de establecer los requisitos mínimos o el establecimiento de normas para iniciar la investigación de nuevos fármacos, algunos puntos deben ser tratados. En primer lugar, no todas las investigaciones que se encuentran en la literatura estudiaron la composición química del propóleo, y se sugiere que las nuevas investigaciones deberían incluir el estudio del propóleo o sus componentes. Sigue estando presente la variabilidad farmacológica de los preparados, ya que es imposible hacer una comparación sistemática de los estudios desde una estandarización universal de la composición del propóleo, debido a que las propiedades biológicas de los propóleos podrían estar relacionadas con su composición química y la posición de sus fuentes botánicas. 
En cuanto al desarrollo de un medicamento extraído de un tipo específico de propóleo, se ha pensado que es posible en principio. Si se trata de estándares basados en principios activos más importantes, que pueden ser sometidos a ensayos clínicos $y$, finalmente, estar registrados. Con la estandarización adecuada, incluso un medicamento con licencia podría ser producido y registrado. Sin embargo, existe un problema legal para la matriculación del propóleo como "sustancia vegetal", ya que es considerado un producto de la abeja, y no un producto a base de hierbas. Con el fin de convertirse en un nuevo fármaco, el propóleo de diferentes regiones no debe utilizarse como una mezcla de todos los componentes, y se cree que los compuestos fenólicos aislados del propó- leo podrían convertirse en clientes potenciales para la medicina moderna. También se han contemplado los efectos sinérgicos de los compuestos individuales, en función de sus concentraciones. Siendo necesario que antes de establecer una estrategia de uso de este producto, es necesario entender en qué condiciones pueden promover la salud.

La eficacia del propóleo debe estar siempre en comparación con parámetros bien establecidos, y los artículos deben incluir controles positivos o negativos en los experimentos. Los ensayos in vitro han aportado nuevos conocimientos sobre los mecanismos de acción del propóleo, y los experimentos in vivo han proporcionado información sobre las propiedades biológicas de este producto.

\section{Conclusiones}

Hay poca información disponible sobre la eficacia clínica del propóleo. Es necesario un nuevo proceso para complementar la investigación básica, la cual servirá para el desarrollo de la investigación clínica, con el fin de evaluar el potencial del propóleo en los pacientes o individuos sanos. Por otro lado, las posibles interacciones entre el propóleo o sus compuestos aislados y otros medicamentos debe ser investigado de igual manera.Esta revisión indica que el propóleo y sus compuestos aislados pueden ser útiles en las ciencias de la salud, siendo un tema de interés que puede ser explotado en el campo de la investigación básica en conjunto con la investigación biomédica.

\section{Bibliografía}

1. Bankov V, Christov R, Kujumgiev A, Marcucci MC, Popov S. Chemical composition and antibacterial activity of Brazilian propolis. Zeitschrift für Naturforschung 1995; 50c, 167-172.

2. Cragg GM, Newman DJ, Snader KM. Natural products in drug discovery and development. Journal of Natural Products 1997; 60:52-60.

3. Greenaway W, Scaysbrook T, Whatley FR. The composition and plant origins of propolis: a report of work at Oxford. Bee World 1990; 71:107-118.
4. Bankova V. Chemical diversity of propolis makes it a valuable source of new biologically active compounds. Journal of Api Product and Api Medical Science 2009; 1, 23-28.

5. Kujumgiev A, Tsvetkova I, Serkedjieva Y, Bankova V, Christov R, Popov S. Antibacterial, antifungal and antiviral activity of propolis of different geographic origin. Journal of Ethnopharmacology1999; 64, 235-240.

6. Trusheva B, Trunkova D, Bankova V.Different extraction methods of biologically active components from propolis: a preliminary study. Chemistry Central Journal 2007; 7, 1-13. 
7. Seidel V, Peyfoon E, Watson DG, Fearnley J. Comparative study of the antibacterial activity of propolis from different geographical and climatic zones. Phytotherapy Research 2008; 22, 1256-1263.

8. Sforcin JM, Fernandes Jr A, Lopes CAM, Bankova $V$, Funari SRC. Season al effect on Brazilian propolis antibacterialactivity. Journal of Ethnopharmacology 2000; 73, 243-249.

9. Búfalo MC, Figueiredo AS, Sousa JPB, Candeias JMG, Bastos JK, Sforcin JM.

Anti-polio virus activity of Baccharisdracunculifolia and propolis by cell viability determination and realtime PCR. Journal of Applied Microbiology 2009c; 107,1669-1680.

10. Heinrich M, Modarai M, Kortenkamp A. Herbal extracts used for upper respiratory tract infections: are there clinically relevant interactions with the cytochrome P450 enzyme system? Planta Médica 2008; 74, 2008.

11. Durán N, Marcato PD, Buffo $C M$, De Azevedo $M M$, Espósito E. Poly (epsilon- caprolactone)/propolisextract: microencapsulation and antibacterial activity evaluation. Pharmazie 2007; 62, 287-290.

12. Kalogeropoulos N, Konteles S, Mourtzinos I, TrouIlidou E, Chiou A, Karathanos VT. Encapsulation of complex extracts in beta-cyclodextrin: an application to propolis ethanolic extract. Journal of Microencapsulation 2009a; 26, 603-613.

13. Roy N, Mondal S, Laskar RA, Basu S, Mandal D, Begum NA. Biogenic synthesis of Au and Ag nanoparticles by Indian propolis and its constituents. Colloids and Surfaces B-Biointerfaces 2010; 76, 317-325

14. Popova M, Chen CN, Chen PY, Huang CY, Bankova $\checkmark$. A validated spectrophotometric method for quantification of prenylated flavanones in Pacific propolis from Taiwan. Phytochemical Analysis 2010 a; 21, 186- 191

15. Cunha IBS, Sawaya ACHF, Caetano FM, Shimizu MT, Marcucci MC, Drezza FT, Povia GS, Carvalho $P O$. Factors that influence the yield and composition of Brazilian propolis extracts. Journal of the Brazilian Chemical Society 2004; 15, 964-970.

16. Bankova V. Recent trends and important developments in propolis research. Evidence-based Complementary and Alternative Medicine 2005b; 2, 29-32.

17. Orsi RO, Funari SRC, Barbattini R, Giovani $C$, Frilli $F$, Sforcin JM, Bankova V. Radionuclides in honey bee propolis (Apis mellifera L.). Bulletin of Environmental
Contamination and Toxicology 2006a; 76, 637-640.

18. Ghisalberti EL. Propolis: A review. Bee World 1979; $60,59-84$.

19. Bankova V, Christov R, Stoev G, Popov S. Determination of phenolics from propolis by capillary gas chromatography. Journal of Chromatography 1992; 607, 150-153.

20. Popova M, Silici S, Kaftanoglu O, Bankova V. Antimicrobial activity of Turkish propolis and its qualitative and quantitative chemical composition. Phytomedicine 2005; 12, 221-228.

21. Hernandez IM, Cuesta-Rubio O, Fernandez MC, Perez AR, De Oca Porto RM, Piccinelli AL, Rastrelli $L$. Studies on the constituents of yellow Cuban propolis: GC-MS determination of triterpenoids and flavonoids. Journal of Agricultural and Food Chemistry 2010; 58, 4725-4730.

22. Popova $M$, Bankova V, Butovska $D$, Petkov V Nikolova-Damyanova B, Sabatini AG, Marcazzan GL, Bogdanov S. Validated methods for $t \mathrm{~h}$ e quantification of biologically active constituents of poplar-type propolis. Phytochemical Analysis 2004; $15,235-240$

23. Silva JFM, Souza MC, Matta SR, Andrade MR, Vidal FVN. Correlation analysis between phenolic levels of Brazilian propolis extracts and their antimicrobial and antioxidant activities. Food Chemistry 2006; 99 431-435.

24. Kumazawa $S$, Nakamura J, Murase $M$, Miyagawa $M$, Ahn MR, Fukumoto S. Plant origin of Okinawan propolis: honey bee behavior observation and phytochemical analysis. Naturwissenschaften 2008; 95 , 781-786.

25. Popova M, Graikou K, Chinou I, Bankova V. GC-MS profiling of diterpene compounds in Mediterranean propolis from Greece. Journal of Agricultural and Food Chemistry 2010b; 58, 3167-3176.

26. Piccinelli AL, Campo Fernandez $M$, Cuesta-Rubio O, Márquez Hernandez I, De Simone F, Rastrelli L. Isoflavonoids isolated from Cuban propolis. Journal of Agricultural and Food Chemistry 2005; 53, 90109016.

27. Lotti C, Campo Fernandez M, Piccinelli AL, CuestaRubio O, Marquez Hernandez I, Rastrelli L. Chemical constituents of red Mexican propolis. Journal of Agricultural and Food Chemistry 2010; 58, 2209-2213. 